This is an author produced version of a paper published in Communications in Statistics - Theory and Methods. This paper has been peer-reviewed and is proof-corrected, but does not include the journal pagination.

Citation for the published paper:

Forkman, J. (2009) Estimator and Tests for Common Coefficients of Variation in Normal Distributions. Communications in Statistics - Theory and Methods. Volume: 38 Number: 2, pp 233-251.

http://dx.doi.org/10.1080/03610920802187448

Access to the published version may require journal subscription.

Published with permission from: Taylor \& Francis

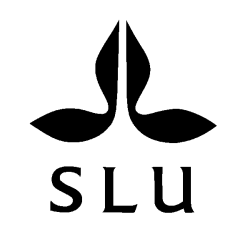

Epsilon Open Archive http://epsilon.slu.se 


\title{
Estimator and Tests for Common Coefficients of Variation in Normal Distributions
}

\author{
Johannes Forkman \\ Department of Energy and Technology \\ Swedish University of Agricultural Sciences \\ Box 7032, SE-750 07 Uppsala, Sweden \\ Johannes.Forkman@vpe.slu.se
}

\begin{abstract}
Inference for the coefficient of variation in normal distributions is considered. An explicit estimator of a coefficient of variation that is shared by several populations with normal distributions is proposed. Methods for making confidence intervals and statistical tests, based on McKay's approximation for the coefficient of variation, are provided. Exact expressions for the first two moments of McKay's approximation are given. An approximate F-test for equality of a coefficient of variation that is shared by several normal distributions and a coefficient of variation that is shared by several other normal distributions is introduced.
\end{abstract}

Key words: Coefficient of Variation, Confidence interval, Hypothesis test, McKay's approximation, Normal distribution, Statistical inference

\section{Introduction}

The coefficient of variation $c$ in a single sample with observations $y_{1}, y_{2}, \ldots, y_{n}$ is defined as $c=s / m$, where $m$ and $s$ are

$$
m=\frac{1}{n} \sum_{j=1}^{n} y_{j} \quad \text { and } \quad s=\sqrt{\frac{1}{n-1} \sum_{j=1}^{n}\left(y_{j}-m\right)^{2}},
$$

respectively. In this paper we consider independently normally distributed observations with expected value $\mu>0$, variance $\sigma^{2}$ and population coefficient of variation $\gamma=\sigma / \mu$. We discuss three problems:

(i) Estimation of a coefficient of variation $\gamma$ that is shared by $k$ populations

(ii) Confidence interval and test for a coefficient of variation $\gamma$ that is shared by $k$ populations 
(iii) Test for equality of a coefficient of variation $\gamma_{1}$ that is shared by $k_{1}$ populations and a coefficient of variation $\gamma_{2}$ that is shared by $k_{2}$ populations

Given $k$ estimates $c_{1}, \ldots, c_{k}$ of a common coefficient of variation $\gamma$ a method is needed for pooling the estimates into one estimate. Explicitly we want to know if the single estimates shall be weighted by the number of observations $n_{i}$, by the degrees of freedom $n_{i}-1$ or by some other function of the sample size.

Zeigler (1973) compared several estimators of a common coefficient of variation, but considered only the case of equally large sample sizes, and did not discuss hypothesis tests and confidence intervals. Tian (2005) studied the problem of making inference about a common $\gamma$ based on $k$ samples and suggested a repeated sampling method for calculating a generalized probability value as defined by Tsui and Weerahandi (1989). A drawback with resampling methods is that they do not give the same result whenever applied. There could be a need for a method based on explicit expressions. Verrill and Johnson (2007) proposed a likelihood ratio based confidence interval for a common coefficient of variation. However, the likelihood ratio test is known to be too liberal for small sample sizes (Doornbos and Dijkstra, 1983; Fung and Tsang, 1998; Nairy and Rao, 2003). This is confirmed in Section 4.2 of the present paper. The likelihood ratio test is computationally inconvenient when there are many populations. Verrill and Johnson (2007) provided, for the likelihood ratio test, a web-based program that simulates critical values for small samples.

We suggest that confidence intervals and tests for a common coefficient of variation $\gamma$ be based on the transformation for the coefficient of variation developed by McKay (1932). This transformation gives an approximately $\chi^{2}$ distributed random variable when $\gamma<1 / 3$, as confirmed by Fieller (1932), Pearson (1932), Iglewicz and Myers (1970) and Umphrey (1983). Forkman and Verrill (2008) showed that McKay's $\chi^{2}$ approximation is asymptotically normal with mean $n-1$ and variance slightly smaller than $2(n-1)$. Vangel (1996) showed, by Taylor series expansion, that the error in McKay's approximation is small when the population coefficient of variation is small. We derive exact expressions for the first two moments of McKay's approximation.

A test is introduced, based on McKay's transformation, for the equality of a coefficient of variation that is shared by $k_{1}$ populations and a coefficient of variation that is shared by $k_{2}$ populations. Many tests have been proposed for the special case $k_{1}=k_{2}=1$ : the likelihood ratio test (Lohrding, 1975; 
Bennett, 1977; Doornbos and Dijkstra, 1983), the Wald test and the score test (Gupta and Ma, 1996), Bennett's test (Bennett, 1976; Shafer and Sullivan, 1986) and Miller's test (Miller, 1991a; Feltz and Miller, 1996; Miller and Feltz, 1997).

The three problems listed above are considered in Sections $2-4$, respectively. In Section 4.2 we make a small Monte Carlo study of the performance of the new test for equality of coefficients of variation compared with the likelihood ratio test, Bennett's test and Miller's test.

\section{Estimation of a Common Coefficient of Varia- tion}

Consider samples from $k$ normally distributed populations with a common population coefficient of variation $\gamma$, and define the sample coefficients of variation as in Definition 1.

Definition 1. Let $y_{i j}=\mu_{i}+e_{i j}$, where $e_{i j}$ are independently distributed $\mathrm{N}\left(0, \sigma_{i}^{2}\right), i=1,2, \ldots, k$ and $j=1,2, \ldots, n_{i}$, with positive expected values $\mu_{i}$ and a positive common population coefficient of variation $\gamma=\sigma_{i} / \mu_{i}$, $i=1,2, \ldots, k$. The coefficient of variation $c_{i}$ of sample $i, i=1,2, \ldots, k$, is defined as $c_{i}=s_{i} / m_{i}$, where $m_{i}$ and $s_{i}$ are

$$
m_{i}=\frac{1}{n_{i}} \sum_{j=1}^{n_{i}} y_{i j} \quad \text { and } \quad s_{i}=\sqrt{\frac{1}{n_{i}-1} \sum_{j=1}^{n_{i}}\left(y_{i j}-m_{i}\right)^{2}},
$$

respectively.

We shall, throughout this paper, assume that the expected values $\mu_{i}$, $i=1,2, \ldots, k$, are positive, which implies $\gamma>0$. Since we are focused on applications with positive and approximately normally distributed observations, we also assume that the probability of negative observations is small. We make this assumption by requiring $\mu_{i}-3 \sigma_{i}>0, i=1,2, \ldots, k$. This implies $\gamma<1 / 3$.

The joint probability density function of the observations $\left\{y_{i j}\right\}$ can be written

$$
\prod_{i=1}^{k}\left((2 \pi)^{-n_{i} / 2} \exp \left(\frac{1}{\mu_{i} \gamma^{2}} \sum_{j=1}^{n_{i}} y_{i j}-\frac{1}{2 \mu_{i}^{2} \gamma^{2}} \sum_{j=1}^{n_{i}} y_{i j}^{2}-\frac{n_{i}}{2 \gamma^{2}}-n_{i} \log \mu_{i} \gamma\right)\right) .
$$


Thus, by the factorization theorem, the $2 k$ dimensional statistic

$$
S=\left\{\sum_{j=1}^{n_{i}} y_{i j}, \sum_{j=1}^{n_{i}} y_{i j}^{2}\right\}_{i=1}^{k}
$$

is sufficient for $\eta=\left\{1 /\left(\mu_{i} \gamma^{2}\right), 1 /\left(\mu_{i}^{2} \gamma^{2}\right)\right\}_{i=1}^{k}$, and since there is a one-to-one correspondence between $\eta$ and $\beta=\left(\gamma, \mu_{1}, \mu_{2}, \ldots, \mu_{k}\right), S$ is also sufficient for $\beta$. By writing (3) as

$$
\exp \left(-\frac{1}{2 \gamma^{2}} \sum_{i=1}^{k} \sum_{j=1}^{n_{i}} \frac{\left(y_{i j}-\mu_{i}\right)^{2}}{\mu_{i}^{2}}-\sum_{i=1}^{k} n_{i} \log \mu_{i} \gamma-\sum_{i=1}^{k} \frac{n_{i}}{2} \log (2 \pi)\right),
$$

we see that

$$
\sum_{i=1}^{k} \sum_{j=1}^{n_{i}} \frac{\left(y_{i j}-\mu_{i}\right)^{2}}{\mu_{i}^{2}}
$$

is complete and sufficient for $\gamma^{2}$, when $\mu_{i}, i=1,2, \ldots, k$, are known. When $\mu_{i}, i=1,2, \ldots, k$, are unknown, $\mu_{i}$ can be estimated by $m_{i}$. Thus, with notation according to Definition 1 , consider

$$
U=\frac{1}{\sum_{i=1}^{k}\left(n_{i}-1\right)} \sum_{i=1}^{k} \sum_{j=1}^{n_{i}} \frac{\left(y_{i j}-m_{i}\right)^{2}}{m_{i}^{2}}=\frac{\sum_{i=1}^{k}\left(n_{i}-1\right) c_{i}^{2}}{\sum_{i=1}^{k}\left(n_{i}-1\right)}
$$

as an estimator of $\gamma^{2}$.

Theorem 1. Let $\gamma$ be a common population coefficient of variation, as defined in Definition 1. Let $v=\sum_{i=1}^{k}\left(n_{i}-1\right)$, and let $U_{v}=U$ as defined by (4). Assume that $\left(n_{i}-1\right) / v \rightarrow \lambda_{i}>0$ as $v \rightarrow \infty$. Then

$$
\sqrt{v}\left(U_{v}-\gamma^{2}\right) \stackrel{d}{\rightarrow} \mathrm{N}\left(0,2 \gamma^{4}+4 \gamma^{6}\right), \quad \text { as } v \rightarrow \infty
$$

Proof. With notations according to Definition 1

$$
\sqrt{n_{i}}\left(m_{i}-\mu_{i}, s_{i}^{2}-\sigma_{i}^{2}\right) \stackrel{d}{\rightarrow} \mathrm{N}(\mathbf{0}, \mathbf{V}), \quad i=1,2, \ldots, k,
$$

where

$$
\mathbf{V}=\left(\begin{array}{cc}
\sigma_{i}^{2} & 0 \\
0 & 2 \sigma_{i}^{4}
\end{array}\right)
$$


Following Serfling (1980, p. 124)

$$
\sqrt{n_{i}}\left(c_{i}^{2}-\gamma^{2}\right) \rightarrow_{d} \mathrm{~N}\left(0, \mathbf{g}^{\prime} \mathbf{V g}\right), \quad i=1,2, \ldots, k,
$$

where, evaluated at $\left\{m_{i}, s_{i}^{2}\right\}=\left\{\mu_{i}, \sigma_{i}^{2}\right\}$,

$$
\mathbf{g}^{\prime}=\left(\frac{\partial c_{i}^{2}}{\partial m_{i}}, \frac{\partial c_{i}^{2}}{\partial s_{i}^{2}}\right)=\left(\frac{-2 \sigma_{i}^{2}}{\mu_{i}^{3}}, \frac{1}{\mu_{i}^{2}}\right) .
$$

Thus

$$
\sqrt{n_{i}}\left(c_{i}^{2}-\gamma^{2}\right) \stackrel{d}{\rightarrow} \mathrm{N}\left(0,2 \gamma^{4}+4 \gamma^{6}\right), \quad i=1,2, \ldots, k,
$$

and, because $\sum_{i=1}^{k} \lambda_{i}=1$,

$$
\sqrt{v}\left(U-\gamma^{2}\right)=\sum_{i=1}^{k} \sqrt{\frac{n_{i}-1}{v}} \sqrt{n_{i}-1}\left(c_{i}^{2}-\gamma^{2}\right) \stackrel{d}{\rightarrow} \mathrm{N}\left(0,2 \gamma^{4}+4 \gamma^{6}\right) . \diamond
$$

We now consider

$$
T=f(U)=\sqrt{U},
$$

with $U$ from (4), as an estimator of $\gamma$. According to Theorem 2 the estimator (5) is asymptotically normally distributed with mean $\gamma$ and variance $\left(\gamma^{2} / 2+\gamma^{4}\right) / \sum_{i=1}^{k}\left(n_{i}-1\right)$.

Theorem 2. Let $\gamma$ be a common population coefficient of variation, as defined by Definition 1 , and let $v=\sum_{i=1}^{k}\left(n_{i}-1\right)$. Let $T_{v}=\sqrt{U_{v}}$, where $U_{v}=U$ as defined by (4). Then

$$
\sqrt{v}\left(T_{v}-\gamma\right) \stackrel{d}{\rightarrow} \mathrm{N}\left(0, \gamma^{2} / 2+\gamma^{4}\right), \quad \text { as } v \rightarrow \infty .
$$

Proof. By Theorem 1, $\sqrt{v} U_{v} \stackrel{d}{\rightarrow} \mathrm{N}\left(\gamma^{2}, 2 \gamma^{4}+4 \gamma^{6}\right)$ as $v \rightarrow \infty$. Then, by application of Theorem 3.1A in Serfling (1980),

$$
\sqrt{v} T_{v}=\sqrt{v} f\left(U_{v}\right) \stackrel{d}{\rightarrow} \mathrm{N}\left(f\left(\gamma^{2}\right),\left(f^{\prime}\left(\gamma^{2}\right)\right)^{2}\left(2 \gamma^{4}+4 \gamma^{6}\right)\right), \quad \text { as } v \rightarrow \infty,
$$

and (6) follows since $\left(f^{\prime}\left(\gamma^{2}\right)\right)^{2}\left(2 \gamma^{4}+4 \gamma^{6}\right)=\gamma^{2} / 2+\gamma^{4}$. $\diamond$

The expected values of the coefficients of variation $c_{i}$ are not defined, because the densities of $m_{i}, i=1,2, \ldots, k$, are positive in neighborhoods of zero. As a consequence the expected value of the estimator $T$, as defined by (5), does not exist. Nevertheless, when the expected values $\mu_{i}$ are sufficiently 
large, say larger than $3 \sigma_{i}$, the probability of averages $m_{i}$ close to zero are negligible in most applications, and we expect $c_{i}, i=1,2, \ldots, k$, to be close to $\gamma$. According to Theorem 2 the estimator $T$ is asymptotically unbiased. We shall now derive a bias correction for the case of small sample sizes.

By a second order series expansion of $T$, as a function of $\left\{m_{i}, s_{i}^{2}\right\}, i=$ $1,2, \ldots, k$,

$$
E(T) \approx \gamma+\frac{1}{2} \sum_{i=1}^{k}\left(\frac{\partial^{2} T}{\partial\left(s_{i}^{2}\right)^{2}} \operatorname{Var}\left(s_{i}^{2}\right)+\frac{\partial^{2} T}{\partial m_{i}^{2}} \operatorname{Var}\left(m_{i}\right)\right),
$$

where the partial second order derivatives should be evaluated at $\left\{m_{i}, s_{i}^{2}\right\}_{i=1}^{k}$ $=\left\{\mu_{i}, \sigma_{i}^{2}\right\}_{i=1}^{k}$. By (7), since $\operatorname{Var}\left(s_{i}^{2}\right)=2 \sigma_{i}^{4} /\left(n_{i}-1\right)$ and $\operatorname{Var}\left(m_{i}\right)=\sigma_{i}^{2} / n_{i}$,

$$
\begin{aligned}
E(T) & \approx \gamma+\frac{1}{2} \sum_{i=1}^{k}\left(\frac{-\left(n_{i}-1\right)^{2}}{4 v^{2} \gamma^{3} \mu_{i}^{4}} \frac{2 \sigma_{i}^{4}}{n_{i}-1}+\frac{\left(n_{i}-1\right) \gamma}{v \mu_{i}^{2}}\left(3-\frac{n_{i}-1}{v}\right) \frac{\sigma_{i}^{2}}{n_{i}}\right) \\
& =\gamma+\frac{1}{2} \sum_{i=1}^{k}\left(\frac{-\left(n_{i}-1\right) \gamma}{2 v^{2}}+\frac{\gamma^{3}}{v} \frac{n_{i}-1}{n_{i}}\left(3-\frac{n_{i}-1}{v}\right)\right) \\
& =\gamma-\frac{\gamma}{4 v}+\frac{\gamma^{3}}{2 v} \sum_{i=1}^{k} \frac{n_{i}-1}{n_{i}}\left(3-\frac{n_{i}-1}{v}\right)
\end{aligned}
$$

where $v=\sum_{i=1}^{k}\left(n_{i}-1\right)$. Thus we expect $T$ to be close to $\gamma(1-1 /(4 v))$ when $\gamma$ is small, and a bias adjusted estimator of a common population coefficient of variation $\gamma$ is given by

$$
\hat{\gamma}=\left(1-\frac{1}{4 \sum_{i=1}^{k}\left(n_{i}-1\right)}\right)^{-1} \sqrt{\frac{\sum_{i=1}^{k}\left(n_{i}-1\right) c_{i}^{2}}{\sum_{i=1}^{k}\left(n_{i}-1\right)}},
$$

with notations as in Definition 1.

The result of a simulation study is presented in Table 1. Three samples of $n_{1}, n_{2}$ and $n_{3}$ observations from normal distributions with expected values $100,1,000$ and 10,000, respectively, and with a common population coefficient of variation $\gamma$, were generated 20,000 times in MATLAB 6.5 (The Mathworks Inc., Natick, MA, USA). In total 18 combinations of $\gamma, n_{1}, n_{2}$ and $n_{3}$ were investigated. For each combination, the means of the unadjusted estimator (5), the bias adjusted estimator (8), and their standard deviation were calculated. The study indicates that the bias adjustment works well for coefficients of variation smaller than $20 \%$. In many chemical applications the 
coefficient of variation is smaller than $20 \%$, and the bias adjusted estimator (8) is then recommended. For example, in immunoassays the working range is often defined as the range of concentrations for which the coefficient of variation is smaller than $20 \%$ (Carroll, 2003).

Table 1: Averages and standard deviations of simulated estimators $T$ and $\hat{\gamma}$ of a common coefficient of variation $\gamma$

\begin{tabular}{crrrccc}
\hline \hline$\gamma$ & $n_{1}$ & $n_{2}$ & $n_{3}$ & $\operatorname{Mean}(T)$ & $\operatorname{Mean}(\hat{\gamma})$ & $\mathrm{SD}$ \\
\hline 0.05 & 2 & 2 & 2 & 0.0461 & 0.0503 & 0.0214 \\
0.05 & 3 & 4 & 5 & 0.0486 & 0.0500 & 0.0119 \\
0.05 & 10 & 10 & 10 & 0.0495 & 0.0500 & 0.0068 \\
\hline 0.10 & 2 & 2 & 2 & 0.0927 & 0.1011 & 0.0430 \\
0.10 & 3 & 4 & 5 & 0.0976 & 0.1004 & 0.0244 \\
0.10 & 10 & 10 & 10 & 0.0994 & 0.1003 & 0.0138 \\
\hline 0.15 & 2 & 2 & 2 & 0.1401 & 0.1528 & 0.0662 \\
0.15 & 3 & 4 & 5 & 0.1470 & 0.1512 & 0.0369 \\
0.15 & 10 & 10 & 10 & 0.1489 & 0.1503 & 0.0210 \\
\hline 0.20 & 2 & 2 & 2 & 0.1887 & 0.2058 & 0.0897 \\
0.20 & 3 & 4 & 5 & 0.1968 & 0.2024 & 0.0501 \\
0.20 & 10 & 10 & 10 & 0.1990 & 0.2009 & 0.0286 \\
\hline 0.25 & 2 & 2 & 2 & 0.2399 & 0.2617 & 0.1184 \\
0.25 & 3 & 4 & 5 & 0.2482 & 0.2553 & 0.0655 \\
0.25 & 10 & 10 & 10 & 0.2496 & 0.2519 & 0.0367 \\
\hline 0.30 & 2 & 2 & 2 & 0.2954 & 0.3222 & 0.1542 \\
0.30 & 3 & 4 & 5 & 0.3005 & 0.3091 & 0.0814 \\
0.30 & 10 & 10 & 10 & 0.3011 & 0.3039 & 0.0458 \\
\hline
\end{tabular}

\section{Confidence Interval and Test for a Common Co- efficient of Variation}

In this section we consider the problems of making a confidence interval for a coefficient of variation $\gamma$ that is shared by $k$ populations and testing the statistical hypothesis that $\gamma$ equals some specific value $\gamma_{0}$. Confidence intervals and tests could be based on the estimator $\hat{\gamma}$ from (8). However, the percentiles of the distribution of $\hat{\gamma}$ are not easily obtained. The unadjusted 
estimator (5) is the square root of a weighted average of squared sample coefficients of variation, and the reciprocal of each sample coefficient of variation is noncentral t-distributed (Owen, 1968). We shall build the confidence interval and test on McKay's approximation, as defined by Definition 2.

Definition 2. McKay's approximation for the coefficient of variation of sample $i$, as defined by Definition 1 , is given by

$$
K_{i}=\left(1+\frac{1}{\gamma^{2}}\right) \frac{\left(n_{i}-1\right) c_{i}^{2}}{1+c_{i}^{2}\left(n_{i}-1\right) / n_{i}} .
$$

The expected value of McKay's approximation (9) exists, and the distribution of (9) is well approximated by a central $\chi^{2}$ distribution with $n_{i}-1$ degrees of freedom, provided that $\gamma<1 / 3$. McKay's approximation is useful for approximately normally distributed measurements of variables that only take positive values. As noted in Section 2, if only positive values can be obtained and the distribution is approximately normal, the expected value $\mu$ cannot be smaller than 3 standard deviations $\sigma$. The requirement $\gamma=\sigma / \mu<1 / 3$, needed for McKay's approximation to be approximately $\chi^{2}$ distributed, is then fulfilled.

The distribution of

$$
u_{i}=\frac{c_{i}^{2}}{1+c_{i}^{2}\left(n_{i}-1\right) / n_{i}}, \quad i=1,2, \ldots, k,
$$

is consequently well approximated by a distribution with expected value

$$
\theta=\frac{\gamma^{2}}{1+\gamma^{2}}=\gamma^{2}-\gamma^{4}+\gamma^{6} \ldots
$$

and variance inversely proportional to $n_{i}-1$. Because the distribution of McKay's approximation $K_{i}$ is, approximately, central $\chi^{2}$ distributed with $n_{i}-1$ degrees of freedom, $\sum_{i=1}^{k} K_{i}$ is, approximately, central $\chi^{2}$ distributed with $\sum_{i=1}^{k}\left(n_{i}-1\right)$ degrees of freedom. Thus, $\sum_{i=1}^{k} K_{i}=\sum_{i=1}^{k}\left(n_{i}-1\right) u_{i} / \theta$ can be used as an approximate pivotal quantity for constructing a confidence interval for $\theta$ as defined by (11). This approximate $100(1-\alpha) \%$ confidence interval for $\theta$ can be written

$$
\left[\frac{\sum_{i=1}^{k}\left(n_{i}-1\right) u_{i}}{\chi_{1-\alpha / 2}^{2}}, \frac{\sum_{i=1}^{k}\left(n_{i}-1\right) u_{i}}{\chi_{\alpha / 2}^{2}}\right],
$$


where $\chi_{\alpha}^{2}$ denotes the $100 \alpha$ :th percentile of a central $\chi^{2}$ distribution with $\sum_{i=1}^{k}\left(n_{i}-1\right)$ degrees of freedom, and $u_{i}$ is defined by (10). The corresponding approximate $100(1-\alpha) \%$ confidence interval for $\gamma$ is

$$
\left[\sqrt{\frac{\sum_{i=1}^{k}\left(n_{i}-1\right) u_{i}}{\chi_{1-\alpha / 2}^{2}-\sum_{i=1}^{k}\left(n_{i}-1\right) u_{i}}}, \sqrt{\frac{\sum_{i=1}^{k}\left(n_{i}-1\right) u_{i}}{\chi_{\alpha / 2}^{2}-\sum_{i=1}^{k}\left(n_{i}-1\right) u_{i}}}\right] .
$$

Consider the statistical null hypothesis $\mathrm{H}_{0}: \gamma=\gamma_{0}$. This hypothesis is equivalent to the hypothesis $\mathrm{H}_{0}: \theta=\theta_{0}$, where $\theta_{0}=\gamma_{0}^{2} /\left(1+\gamma_{0}^{2}\right)$. Thus we can use

$$
\frac{\sum_{i=1}^{k}\left(n_{i}-1\right) u_{i}}{\theta_{0}}
$$

as an approximately central $\chi^{2}$ distributed, with $\sum_{i=1}^{k}\left(n_{i}-1\right)$ degrees of freedom, test statistic of the hypothesis $\mathrm{H}_{0}: \gamma=\gamma_{0}$.

The proposed confidence interval (12) and test (13) rely on the adequacy of McKay's approximation. Since we are interested in the adequacy, we end this section with an investigation of the first two moments of the approximation, as functions of the population coefficient of variation $\gamma$ and the sample size $n$.

Theorem 4. Let $\gamma$ be the population coefficient of variation as defined by Definition 1, and let $K=K_{1}$ be McKay's approximation for the coefficient of variation in a sample with $n=n_{1}$ observations, as defined by Definition 2 . The first and second moments of McKay's approximation are

$$
\begin{aligned}
E(K) & =\frac{n(n-1) h(\gamma, n)}{2 \theta}, \\
E\left(K^{2}\right) & =\frac{n\left(n^{2}-1\right)\left(n\left(1+\gamma^{2}\right) h(\gamma, n)-2 \gamma^{2}\right)}{4 \theta^{2}},
\end{aligned}
$$

where $\theta=\gamma^{2} /\left(1+\gamma^{2}\right)$ and, for $t=0,1,2 \ldots$,

$$
h(\gamma, n)=
$$




$$
\begin{cases}\gamma^{2}\left(1-\exp \left(-1 / \gamma^{2}\right)\right), & n=2 \\ \sum_{r=0}^{t}(-1)^{r}\left(\frac{\gamma^{2}}{t+3 / 2}\right)^{r+1} \frac{\Gamma(t+3 / 2)}{\Gamma(t+3 / 2-r)} & \\ +(-1)^{t+1}\left(\frac{\gamma^{2}}{t+3 / 2}\right)^{t+3 / 2} \frac{2 \Gamma(t+3 / 2)}{\sqrt{\pi}} d(\sqrt{(t+3 / 2)} / \gamma), & n=3+2 t \\ \sum_{r=0}^{t}(-1)^{r}\left(\frac{\gamma^{2}}{t+2}\right)^{r+1} \frac{\Gamma(t+2)}{\Gamma(t+2-r)} & \\ +(-1)^{t+1}\left(\frac{\gamma^{2}}{t+2}\right)^{t+2} \Gamma(t+2)\left(1-\exp \left(-(t+2) / \gamma^{2}\right)\right), & n=4+2 t\end{cases}
$$

with

$$
d(x)=\exp \left(-x^{2}\right) \int_{0}^{x} \exp \left(z^{2}\right) d z .
$$

Proof. Forkman and Verrill (2008) showed that $K \theta / n$ is type II noncentral beta distributed with parameters $(n-1) / 2,1 / 2$ and $n / \gamma^{2}$. Consequently $X=$ $1-K \theta / n$ is type I noncentral beta distributed with parameters $1 / 2,(n-1) / 2$ and $n / \gamma^{2}$. A type I noncentral beta distribution with parameters $a, b$ and $\delta$ is central $\operatorname{Beta}(a+V, b)$, where $V$ is $\operatorname{Poisson}(\delta / 2)$. Marchand (1997) provided expressions for the first and second moments of the type I noncentral beta distribution with parameters $a, b$ and $\delta$ based on $g(a+b, \delta / 2)=E(1 /(a+b+$ $V))$. We let $h(\gamma, n)=g\left(n / 2, n /\left(2 \gamma^{2}\right)\right)$. Then, according to Marchand (1997),

$$
\begin{gathered}
E(X)=1-\frac{(n-1) h(\gamma, n)}{2}, \\
E\left(X^{2}\right)=1-\frac{\left(n^{2}-1\right) \gamma^{2}}{2 n}+\frac{(n-1)\left(n-3+(n+1) \gamma^{2}\right) h(\gamma, n)}{4},
\end{gathered}
$$

and the theorem follows since $E(K)=n(1-E(X)) / \theta$ and $E\left(K^{2}\right)=n^{2}(1-$ $\left.2 E(X)+E\left(X^{2}\right)\right) / \theta^{2}$.

The function $d$, required for odd sample sizes in Theorem 4, is Dawsons's integral, which has been tabulated by Abramowitz and Stegun (1972). Because $\exp \left(-(t+2) / \gamma^{2}\right) \approx 0, t=0,1,2 \ldots$, Theorem 4 makes it easy to calculate approximate first and second moments, especially for even sample 
sizes. For example,

$$
E(K) \approx \begin{cases}1\left(1+\gamma^{2}\right), & n=2 \\ 3\left(1+\frac{\gamma^{2}}{2}-\frac{\gamma^{4}}{2}\right), & n=4 \\ 5\left(1+\frac{\gamma^{2}}{3}+\frac{2 \gamma^{4}}{9}+\frac{8 \gamma^{6}}{9}\right), & n=6,\end{cases}
$$

and

$$
E\left(K^{2}\right) \approx \begin{cases}3\left(1+2 \gamma^{2}+\gamma^{4}\right), & n=2 \\ 15\left(1+\gamma^{2}-\gamma^{4}-\gamma^{6}\right), & n=4 \\ 35\left(1+\frac{8 \gamma^{2}}{3}+5 \gamma^{4}+6 \gamma^{6}+\frac{8 \gamma^{8}}{3}\right), & n=6 .\end{cases}
$$

Notice that when $\gamma$ is small, $n=2,4,6, E(K)$ approximately equals $n-1$ and $E\left(K^{2}\right)$ approximately equals $(n-1)(n+1)$, which is the exact first and second moments, respectively, of a $\chi^{2}$ distributed random variable with $n-1$ degrees of freedom.

\section{Test for Equality of Two Common Coefficients of Variation}

We now introduce a statistical test for the hypothesis that a coefficient of variation $\gamma_{1}$ that is shared by $k_{1}$ populations equals a coefficient of variation $\gamma_{2}$ that is shared by $k_{2}$ populations. Definition 3 makes clear the setting and what we mean by coefficients of variation in this case.

Definition 3. Let $y_{r i j}=\mu_{r i}+e_{r i j}$, where $e_{r i j}$ are independently distributed $\mathrm{N}\left(0, \sigma_{r i}^{2}\right), r=1,2, i=1,2, \ldots, k_{r}$ and $j=1,2, \ldots, n_{r i}$, with positive expected values $\mu_{r i}$ and positive population coefficients of variation $\gamma_{r}=\sigma_{r i} / \mu_{r i}$. The coefficient of variation $c_{r i}, r=1,2, i=1,2, \ldots, k_{r}$, is defined as $c_{r i}=s_{r i} / m_{r i}$, where $m_{r i}$ and $s_{r i}$ are

$$
m_{r i}=\frac{1}{n_{r i}} \sum_{j=1}^{n_{r i}} y_{r i j} \quad \text { and } \quad s_{r i}=\sqrt{\frac{1}{n_{r i}-1} \sum_{j=1}^{n_{r i}}\left(y_{r i j}-m_{r i}\right)^{2}}
$$


respectively.

\subsection{A Test for Equality of Coefficients of Variation}

Consider the hypothesis $\mathrm{H}_{0}: \gamma_{1}=\gamma_{2}$. Let

$$
u_{r i}=\frac{c_{r i}^{2}}{1+c_{r i}^{2}\left(n_{r i}-1\right) / n_{r i}}, \quad r=1,2 ; i=1,2, \ldots, k_{r},
$$

and

$$
\theta_{r}=\frac{\gamma_{r}^{2}}{1+\gamma_{r}^{2}}, \quad r=1,2,
$$

with notation according to Definition 3. Because $\sum_{i=1}^{k_{r}}\left(n_{r i}-1\right) u_{r i} / \theta_{r}$ is approximately central $\chi^{2}$ distributed with $\sum_{i=1}^{k_{r}}\left(n_{r i}-1\right)$ degrees of freedom, and $\theta_{1}=\theta_{2}$ when the hypothesis is true,

$$
F=\frac{\sum_{i=1}^{k_{1}}\left(n_{1 i}-1\right) u_{1 i} / \sum_{i=1}^{k_{1}}\left(n_{1 i}-1\right)}{\sum_{i=1}^{k_{2}}\left(n_{2 i}-1\right) u_{2 i} / \sum_{i=1}^{k_{2}}\left(n_{2 i}-1\right)}
$$

is approximately $\mathrm{F}$ distributed with $\sum_{i=1}^{k_{1}}\left(n_{1 i}-1\right)$ and $\sum_{i=1}^{k_{2}}\left(n_{2 i}-1\right)$ degrees of freedom. Thus $F$ can be used as an approximately $\mathrm{F}$ distributed test statistic for the hypothesis of equal coefficients of variation.

When $k_{1}=k_{2}=1$, the test statistic $F$, as defined by (17), simplifies to

$$
F=\frac{c_{1}^{2} /\left(1+c_{1}^{2}\left(n_{1}-1\right) / n_{1}\right)}{c_{2}^{2} /\left(1+c_{2}^{2}\left(n_{2}-1\right) / n_{2}\right)},
$$

where $c_{r}=c_{r 1}$ and $n_{r}=n_{r 1}, \mathrm{r}=1,2$, as defined by Definition 3. According to Theorem 6 the distribution of the logarithm of $F$, as defined by (18), equals the distribution of the logarithm of an $\mathrm{F}$ distributed random variable plus some error variables that are in probability of small orders. Let $O_{p}$ denote order in probability, defined as in Azzalini (1996).

Theorem 6. Let $\gamma=\gamma_{1}=\gamma_{2}$ as defined by Definition 3, with $k_{1}=k_{2}=1$. Let $X$ be an $\mathrm{F}$ distributed random variable with $n_{1}-1$ and $n_{2}-1$ degrees of freedom, let $Z$ be a standardized normal random variable, and let $U_{1}$ and $U_{2}$ be $\chi^{2}$ distributed random variables with $n_{1}-1$ and $n_{2}-1$ degrees of freedom, respectively. Let $X, Z, U_{1}$ and $U_{2}$ be independent. Then

$$
\log F \stackrel{d}{=} \log X+2 Z \gamma \sqrt{\frac{1}{n_{1}}+\frac{1}{n_{2}}}+\left(\frac{U_{1}}{n_{1}}-\frac{U_{2}}{n_{2}}\right) \gamma^{2}+R\left(n_{1}, n_{2}, \gamma\right)
$$


where $F$ is defined by (18) and $R\left(n_{1}, n_{2}, \gamma\right)=O_{p}\left(\max \left\{n_{1}^{-1} \gamma^{2}, n_{2}^{-1} \gamma^{2}, \gamma^{4}\right\}\right)$.

Proof. Write $\log F$ as

$$
\log F=\log c_{1}^{2}\left(1+\frac{\left(n_{1}-1\right) c_{1}^{2}}{n_{1}}\right)^{-1}-\log c_{2}^{2}\left(1+\frac{\left(n_{2}-1\right) c_{2}^{2}}{n_{2}}\right)^{-1} .
$$

Let $W_{r}=U_{r} /\left(n_{r}-1\right), r=1,2$, and let $Z_{1}$ and $Z_{2}$ be independent standardized normal random variables. The distributions of the averages $m_{r 1}$ and the standard deviations $s_{r 1}, r=1,2$, as defined by Definition 3, equals the distributions of $\mu_{r 1}+Z_{r} \sigma_{r 1} / \sqrt{n_{r}}$ and $\mu_{r 1} \gamma \sqrt{W_{r}}$, respectively. Thus $c_{r}^{2}$ equals $W_{r} \gamma^{2} /\left(1+Z_{r} \gamma / \sqrt{n}\right)^{2}$ in distribution. The distribution of the first term in (20) consequently equals the distribution of

$$
\begin{array}{r}
\log W_{1} \gamma^{2}-\log \left(1+\frac{2 Z_{1} \gamma}{\sqrt{n_{1}}}+\frac{Z_{1}^{2} \gamma^{2}}{n_{1}}+\frac{\left(n_{1}-1\right) W_{1} \gamma^{2}}{n_{1}}\right) \\
=\log W_{1} \gamma^{2}+\frac{2 Z_{1} \gamma}{\sqrt{n_{1}}}+\frac{Z_{1}^{2} \gamma^{2}}{n_{1}}+\frac{\left(n_{1}-1\right) W_{1} \gamma^{2}}{n_{1}} \\
-\frac{1}{2}\left(\frac{2 Z_{1} \gamma}{\sqrt{n_{1}}}+\frac{Z_{1}^{2} \gamma^{2}}{n_{1}}+\frac{\left(n_{1}-1\right) W_{1} \gamma^{2}}{n_{1}}\right)^{2}+\ldots \\
=\log W_{1} \gamma^{2}+\frac{2 Z_{1} \gamma}{\sqrt{n_{1}}}+\frac{\left(n_{1}-1\right) W_{1} \gamma^{2}}{n_{1}}+O_{p}\left(\max \left(\frac{\gamma^{2}}{n_{1}}, \gamma^{4}\right)\right) .
\end{array}
$$

The corresponding calculations can be made also for the second term in (20), and (19) follows. $\diamond$

\subsection{Simulation Study}

In this section we investigate, by Monte Carlo technique, the significance levels and powers of the introduced approximate F-test (18), for the hypothesis $\mathrm{H}_{0}: \gamma_{1}=\gamma_{2}$ when $k_{1}=k_{2}=1$. We also study the likelihood ratio test, Bennett's test as modified by Shafer and Sullivan (1986), and Miller's test. These tests are, for quick reference, given in the Appendix.

In each simulation two samples with $n_{1}$ and $n_{2}$ observations, respectively, were randomly generated 20,000 times in MATLAB 6.5. The observations were normally distributed with expected values 100 and 1,000, and with coefficients of variation $\gamma_{1}$ and $\gamma_{2}$, respectively. The tests were performed with significance level $5 \%$ against the alternative hypothesis of unequal coefficients of variation, that is the tests were two-sided.

Eight cases were studied. The type I errors of the tests were investigated 
in Cases 1-6, and the powers of the tests were investigated in Cases 7 and 8. In Cases 1-3, the common coefficient of variation $\gamma$ was $5 \%, 15 \%$ and $30 \%$, respectively. The sample sizes were equal, i.e. $n_{1}=n_{2}=n$, and varied varied from 2 to 20 . In Cases $4-6, \gamma$ was $5 \%, 15 \%$ and $30 \%$, respectively, but in these cases $n_{1}=4$ and $n_{2}$ varied from 2 to 20 . In Case 7 one coefficient of variation was $5 \%$, and the other $15 \%$, and in Case 8 one coefficient of variation was $15 \%$, and the other $30 \%$. In Case 7 and 8 , the two sample sizes were equal and varied from 2 to 20 . Thus 19 simulations were made per case.

The results of the simulation study are presented in Figures $1-8$, with one figure per investigated case. The likelihood ratio test showed too large frequency of type I error (Figures 1-6), especially for sample sizes smaller than 10. When $n_{2}=2$ the frequency of rejected hypotheses was larger than $20 \%$ with the likelihood ratio test. Bennett's test was also too liberal, though not as liberal as the likelihood ratio test (Figures 1-6). Miller's test performed better with regard to type I error, except when only 2 observations were sampled per distribution (Figures 1-3). The approximate F-test, introduced in this paper, was the only test that produced almost correct frequency of rejected hypotheses in all cases (Figures 1-6). The likelihood ratio test and Bennett's test, which were too liberal, showed better power for small sample sizes than Miller's test and the approximate F-test (Figures 7 and 8).

\section{Discussion}

In applications with constant, or almost constant, coefficients of variation it is often appropriate to assume that the data follows lognormal distributions. One should therefore consider working on the log scale (Cole, 2000). After log transformation of the data the usual tests for equality of variances, such as Bartlett's test, could be applied.

However, it is not always appropriate to assume that the data is lognormally distributed. In immunoassays, for example, normally distributed errors in the volumes of samples could result in normally distributed measurements of concentration with constant coefficient of variation. In this paper we have discussed inference for the coefficient of variation when there are reasons to believe that the data is normally, but not lognormally, distributed. 

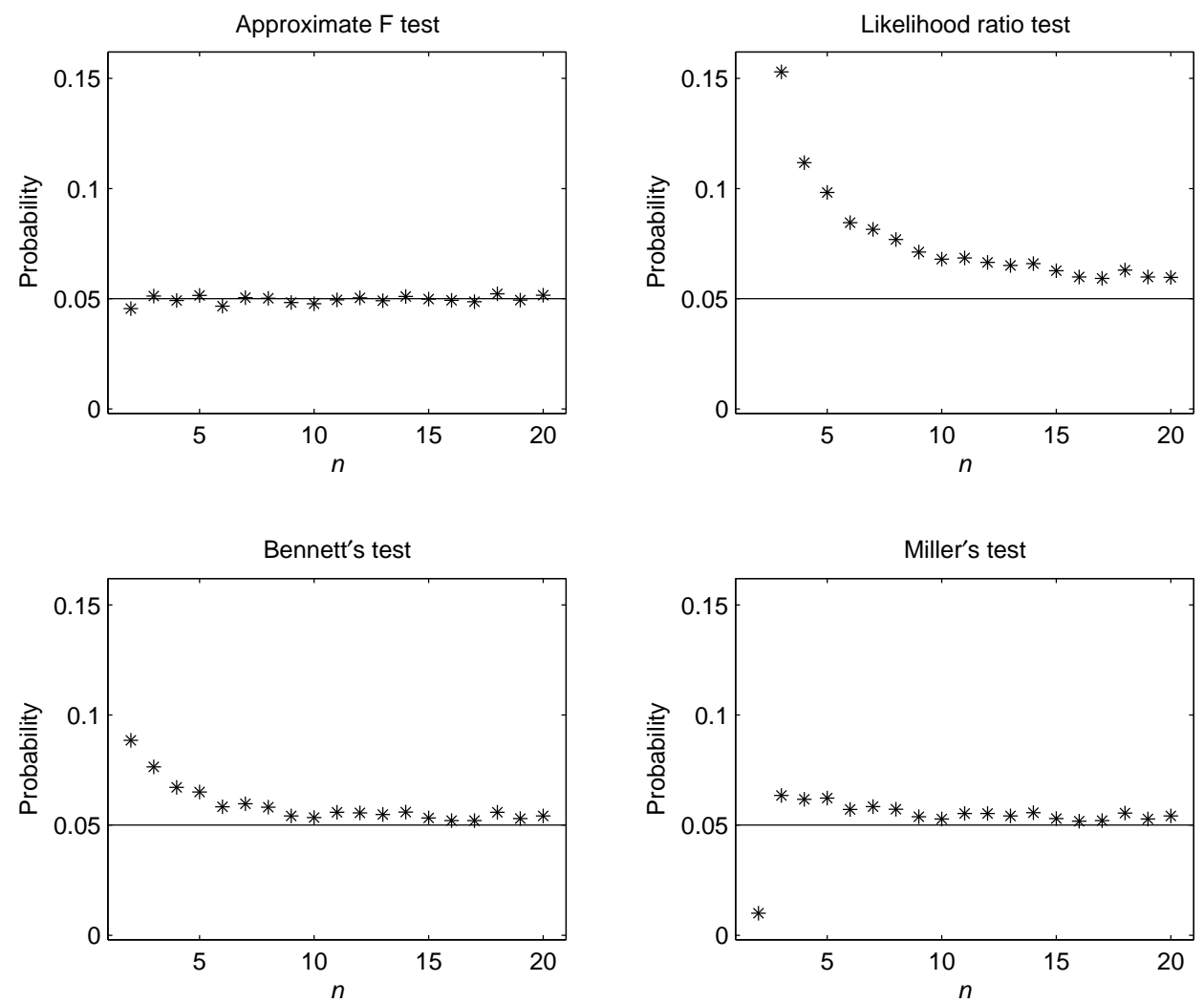

Figure 1: Case 1. Probability of type I error when $\gamma_{1}=\gamma_{2}=5 \%$ and $n=n_{1}=n_{2}$. 

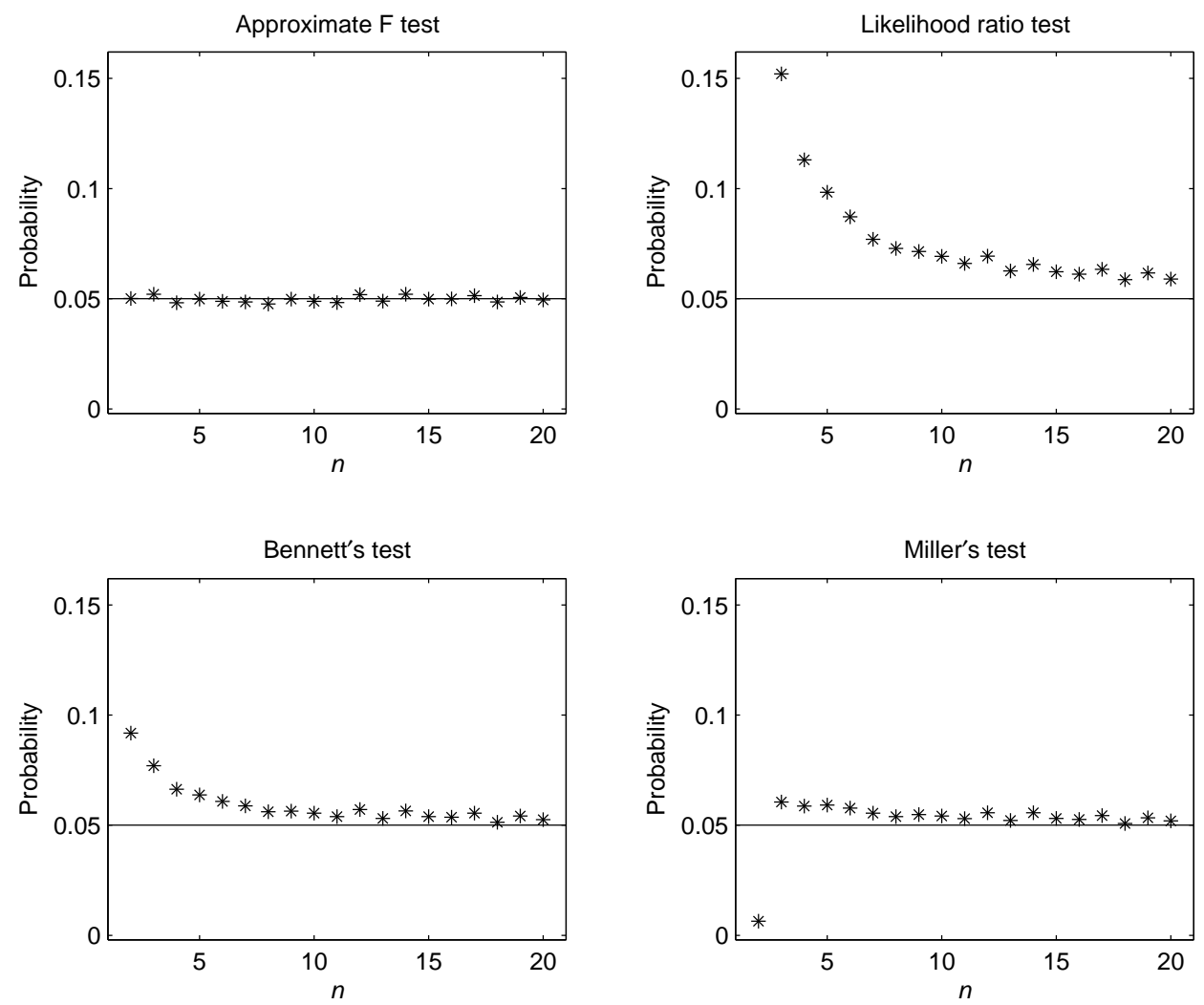

Figure 2: Case 2. Probability of type I error when $\gamma_{1}=\gamma_{2}=15 \%$ and $n=n_{1}=n_{2}$. 

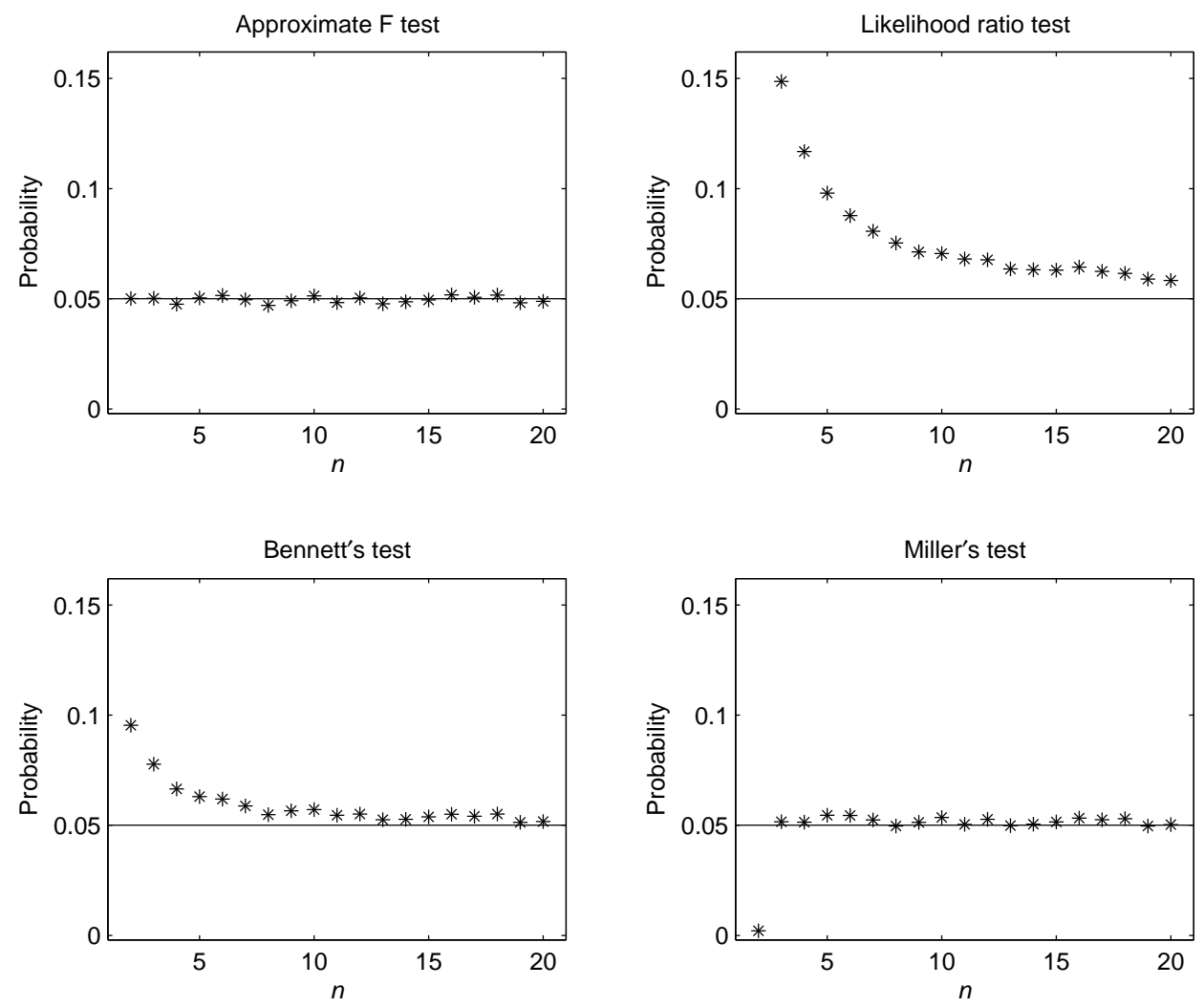

Figure 3: Case 3. Probability of type I error when $\gamma_{1}=\gamma_{2}=30 \%$ and $n=n_{1}=n_{2}$. 

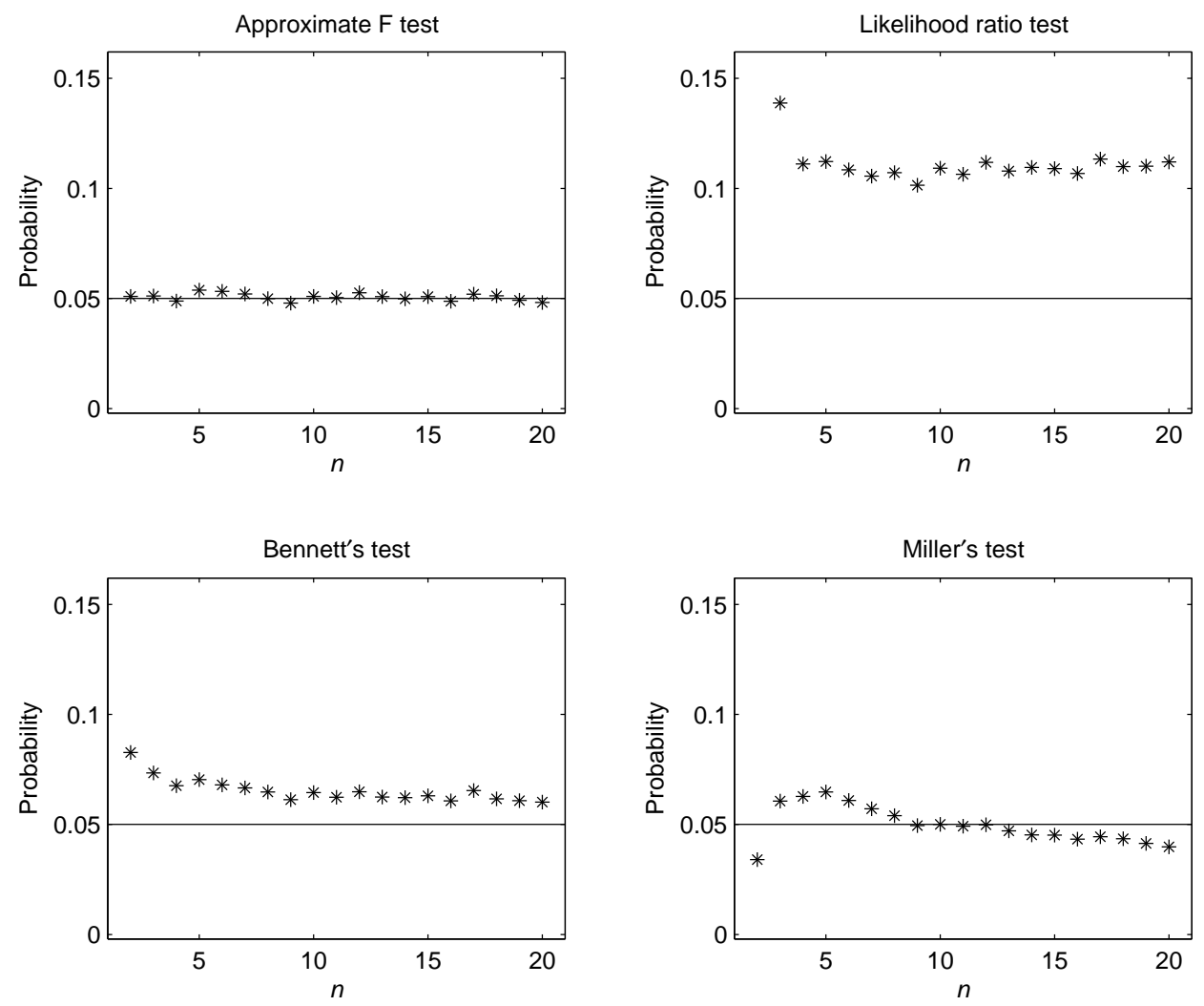

Figure 4: Case 4. Probability of type I error when $\gamma_{1}=\gamma_{2}=5 \%$ and $n_{1}=4$. 

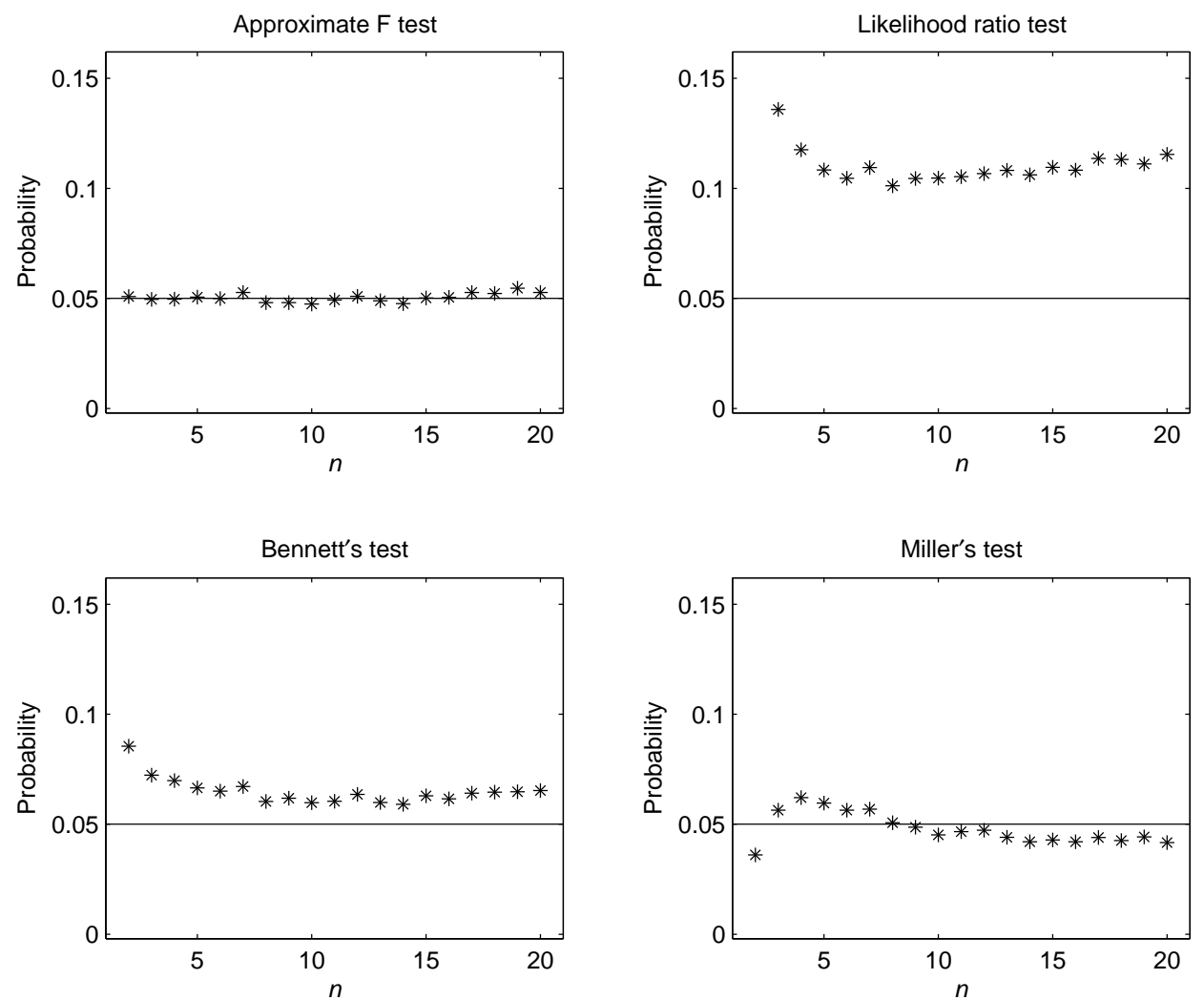

Figure 5: Case 5. Probability of type I error when $\gamma_{1}=\gamma_{2}=15 \%$ and $n_{1}=4$. 

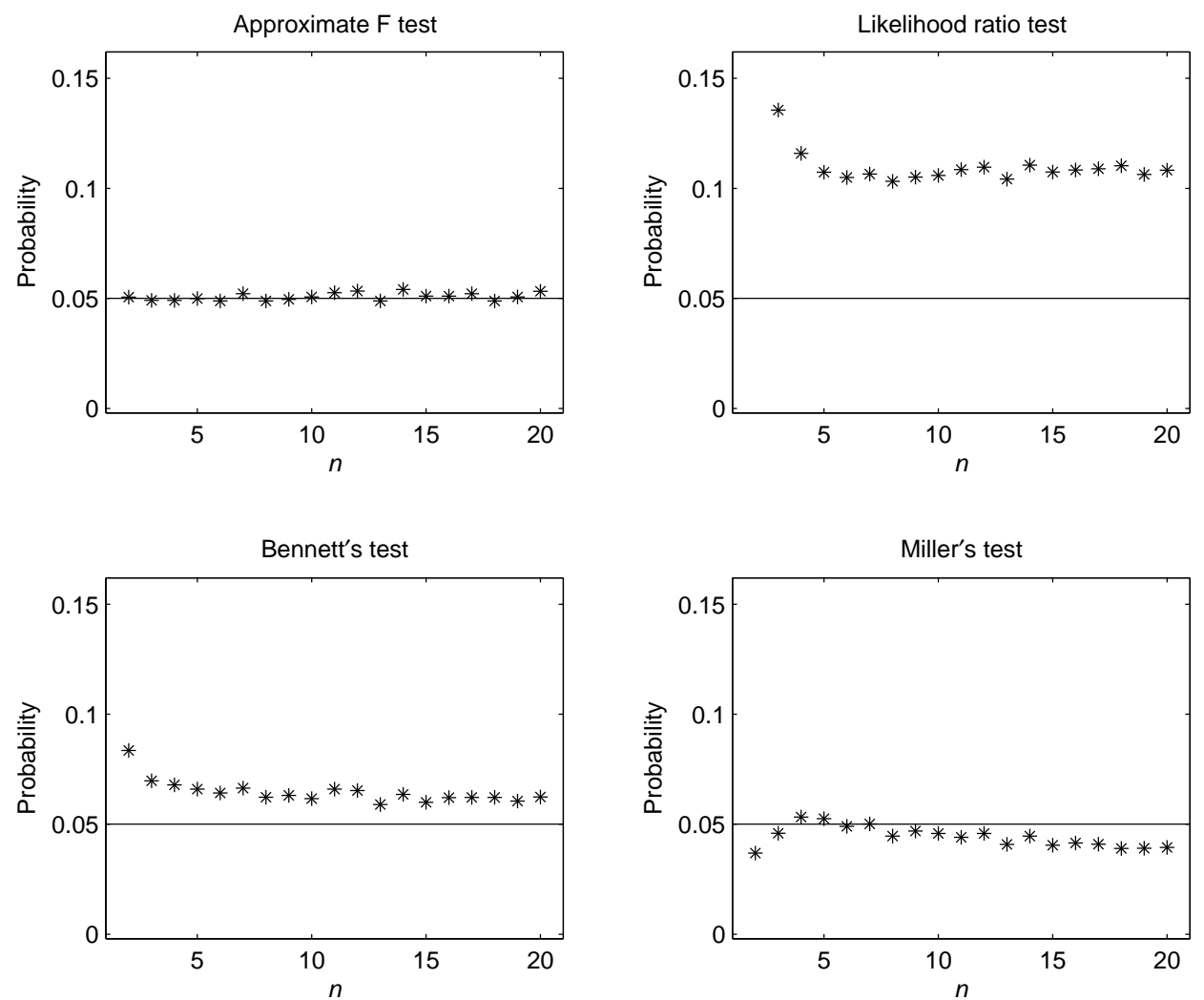

Figure 6: Case 6. Probability of type I error when $\gamma_{1}=\gamma_{2}=30 \%$ and $n_{1}=4$. 

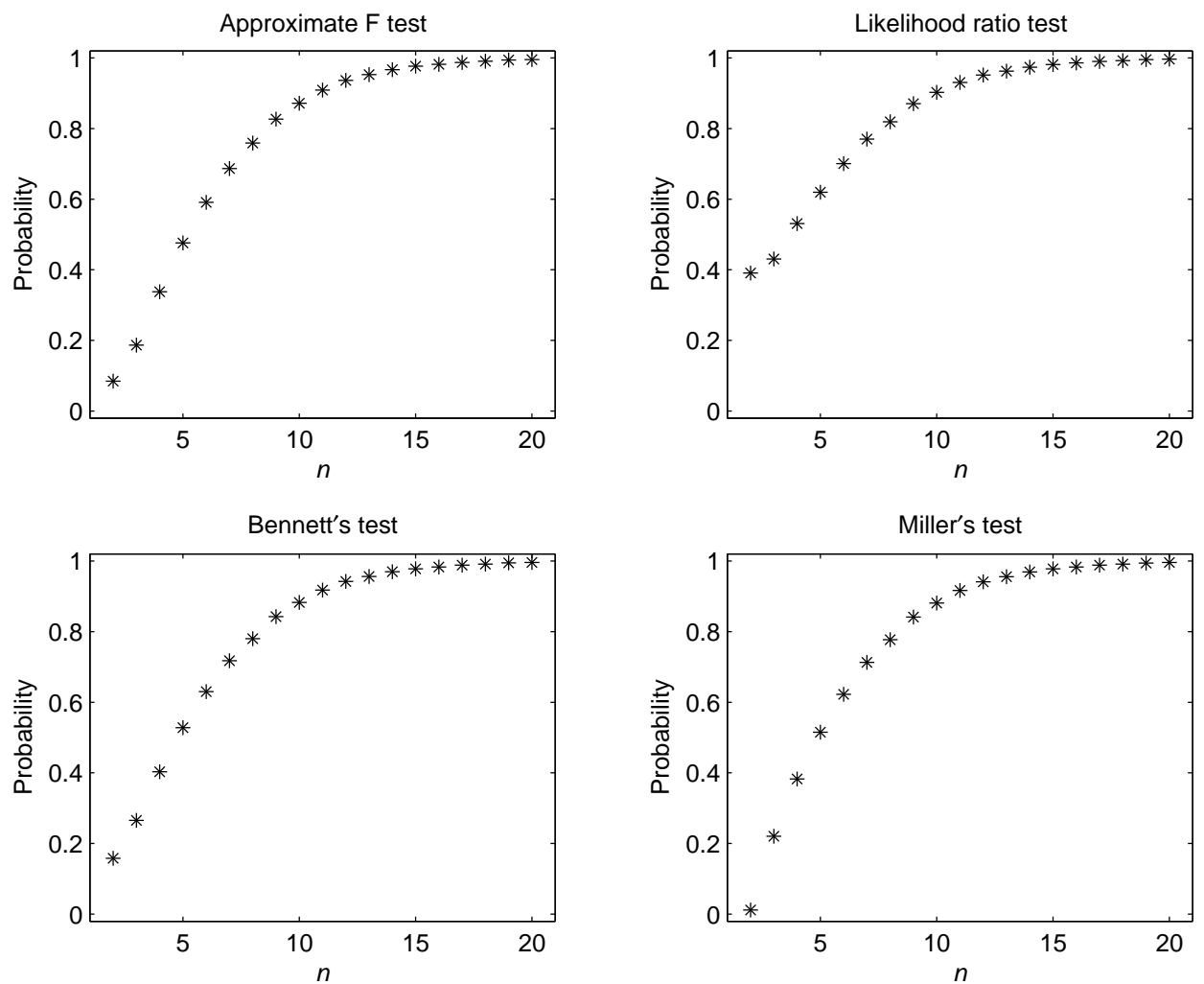

Figure 7: Case 7. Power when $\gamma_{1}=5 \%, \gamma_{2}=15 \%$ and $n=n_{1}=n_{2}$. 

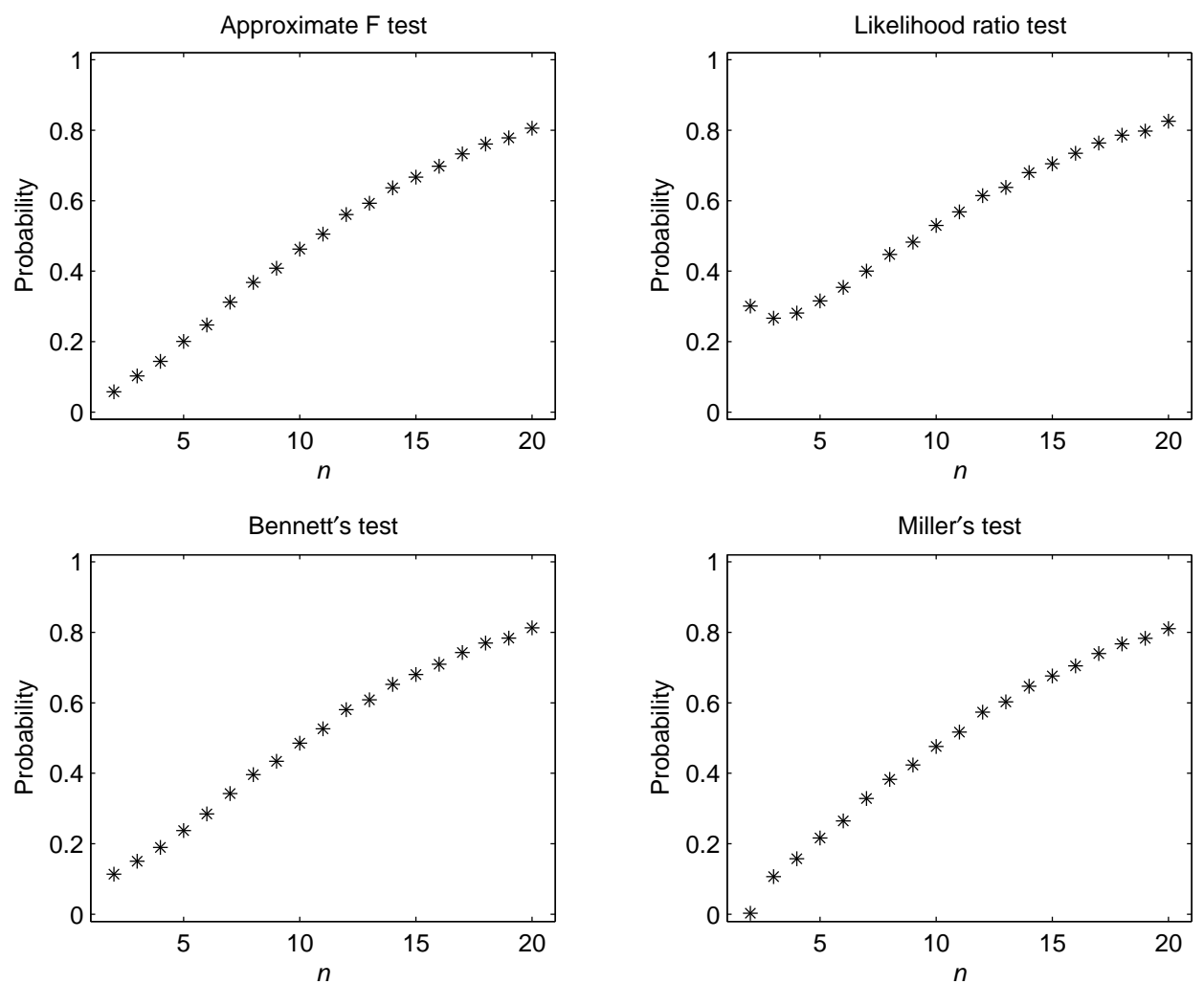

Figure 8: Case 8. Power when $\gamma_{1}=15 \%, \gamma_{2}=30 \%$ and $n=n_{1}=n_{2}$. 
Since it is often necessary to relate the standard deviation to the level of the measurements, the coefficient of variation is a widely used measure of dispersion. Coefficients of variation are often calculated on samples from several independent populations, and questions about how to compare them naturally arise. There is a need for an explicit estimator of a common coefficient of variation. Such an estimator has been given in this paper.

For making confidence intervals and tests we have considered McKay's approximation, which is valid only when the population coefficient of variation $\gamma$ is smaller than $1 / 3$. Coefficients of variation are usually calculated on positive data, such as measurements of concentration, weight or length. Given that the positive measurements are approximately normally distributed $\gamma$ is smaller than $1 / 3$, because otherwise the expected value is smaller than 3 standard deviations, and the probability of negative observations is not negligible. Thus, McKay's approximation is applicable for positive variables, provided only that the distributions are approximately normally distributed. Normality could be checked, e.g. by the Shapiro-Wilk test.

Over the years many tests have been proposed for equality of coefficients of variation. In this paper, an additional test has been introduced: the approximate F-test. Unlike many other tests the new test can be applied not only when there are one estimate per population coefficient of variation, but also when there are several. The small simulation study reported in this paper indicated good performance of the approximate F-test, especially with regard to type I error. It would, however, be interesting to see results from a larger simulation study, including more cases, several tests and an investigation of robustness. As already pointed out, the methods proposed in this paper are intended for normally distributed data. Miller (1991b) suggested a nonparametric test for equality of coefficients of variation. This nonparametric test was recommended for nonnormal distributions by Fung and Tsang (1998).

The $\mathrm{F}_{\text {max }}$-test (Hartley, 1950) is a natural extension of the approximate F-test to more than two common coefficients of variation (G.E. Miller, personal communication). For two coefficients of variation, the $\mathrm{F}_{\text {max }}$-test and the approximate F-test are identical for the two-sided hypothesis. The $\mathrm{F}_{\text {max }}{ }^{-}$ test requires equal degrees of freedom. Tables for the $\mathrm{F}_{\text {max }}$ distribution were given by Nelson (1987). 


\section{Acknowledgments}

I thank the referees for useful comments. The Centre of Biostochastics, Swedish University of Agricultural Sciences, and Pharmacia Diagnostics AB, Uppsala, Sweden, supported the research.

\section{Appendix. The Likelihood ratio test, Bennett's and Miller's Tests}

Let $m_{r}=m_{r 1}$ and $c_{r}=c_{r 1}$ denote the average and the coefficient of variation, respectively, in the $r$ :th sample, $r=1,2$, as defined by Definition 3 . The likelihood ratio test statistic can be written

$$
-2 \log \lambda=n_{1} \log \frac{n_{1}\left(\tilde{\gamma} \tilde{\mu}_{1}\right)^{2}}{\left(n_{1}-1\right) c_{1}^{2} m_{1}^{2}}+n_{2} \log \frac{n_{2}\left(\tilde{\gamma} \tilde{\mu_{2}}\right)^{2}}{\left(n_{2}-1\right) c_{2}^{2} m_{2}^{2}},
$$

where $\lambda$ is the likelihood ratio and $\tilde{\gamma}, \tilde{\mu_{1}}$ and $\tilde{\mu_{2}}$ are the maximum likelihood estimates of $\gamma, \mu_{1}$ and $\mu_{2}$, respectively. These are, according to Gerig and Sen (1980),

$$
\begin{gathered}
\tilde{\mu}_{1}=\frac{n_{1} m_{1} \tilde{\mu}_{2}}{\left(n_{1}+n_{2}\right) \tilde{\mu}_{2}-n_{2} m_{2}}, \quad \tilde{\mu}_{2}=-\frac{q}{2 p}+\sqrt{\frac{q^{2}}{4 p^{2}}-\frac{r}{p}}, \\
\tilde{\gamma}=\frac{1}{\tilde{\mu}_{2}} \sqrt{\frac{\left(n_{2}-1\right) c_{2}^{2} m_{2}^{2}}{n_{2}}+m_{2}^{2}-m_{2} \tilde{\mu}_{2}},
\end{gathered}
$$

where $p=\left(n_{1}+n_{2}\right) c_{1}^{2}+n_{2}, q=-\left(2 n_{2} c_{1}^{2}+2 n_{2}-n_{1}\right) m_{2}$ and $r=\left(\left(n_{2}^{2}\left(c_{1}^{2}+\right.\right.\right.$ $\left.\left.1)-n_{1}^{2}\left(c_{2}^{2}+1\right)\right) m_{2}^{2}\right) /\left(n_{1}+n_{2}\right)$. Asymptotically $(22)$ is $\chi^{2}$ distributed with 1 degree of freedom.

Bennett's test statistic, as modified by Shafer and Sullivan (1986), can be written

$$
\begin{aligned}
\left(n_{1}+n_{2}-2\right) \log \left(\frac{1}{n_{1}+n_{2}-2}\right. & \left.\left(\frac{\left(n_{1}-1\right) c_{1}^{2}}{1+c_{1}^{2}\left(n_{1}-1\right) / n_{1}}+\frac{\left(n_{1}-1\right) c_{1}^{2}}{1+c_{1}^{2}\left(n_{1}-1\right) / n_{1}}\right)\right) \\
& -\left(n_{1}-1\right) \log \left(\frac{\left(n_{1}-1\right) c_{1}^{2}}{\left(n_{1}-1\right)\left(1+c_{1}^{2}\left(n_{1}-1\right) / n_{1}\right.}\right) \\
- & \left(n_{2}-1\right) \log \left(\frac{\left(n_{2}-1\right) c_{2}^{2}}{\left(n_{2}-1\right)\left(1+c_{2}^{2}\left(n_{2}-1\right) / n_{2}\right)}\right),
\end{aligned}
$$

and is approximately $\chi^{2}$ distributed with 1 degree of freedom. 
Miller's test statistic (Miller, 1991a), which is approximately $\mathrm{N}(0,1)$, is

$$
\left(c_{1}-c_{2}\right)\left(\frac{c^{2}}{2\left(n_{1}-1\right)}+\frac{c^{4}}{n_{1}-1}+\frac{c^{2}}{2\left(n_{2}-1\right)}+\frac{c^{4}}{n_{2}-1}\right)^{-1 / 2},
$$

where $c=\left(\left(n_{1}-1\right) c_{1}+\left(n_{2}-1\right) c_{2}\right) /\left(n_{1}+n_{2}-2\right)$.

\section{References}

Abramowitz, M. and Stegun, I. A. (1972). Handbook of Mathematical Functions with Formulas, Graphs, and Mathematical Tables. New York: Dover.

Azzalini, A. (1996). Statistical Inference. London: Chapman and Hall.

Bennett, B. M. (1976). On an approximate test for homogeneity of coefficients of variation. In W. J. Ziegler (Ed.), Contributions to Applied Statistics dedicated to A. Linder. Experientia Supplementum 22:169171. Basel: Birkhäuser.

Bennett, B. M. (1977). LR tests for homogeneity of coefficients of variation in repeated samples. Sankhya, Series B, 39:400-405.

Carroll, R. J. (2003). Variances are not always nuisance parameters. Biometrics 59:211-220.

Cole, T. J. (2000). Sympercents: symmetric percentage differences on the $100 \log _{\mathrm{e}}$ scale simplify the presentation of log transformed data. Statistics in Medicine 19:3109-3125.

Doornbos R. and Dijkstra J. B. (1983). A multi sample test for the equality of coefficients of variation in normal populations. Communications in Statistics - Simulation and Computation 12:147-158.

Feltz, C. J. and Miller G. E (1996). An asymptotic test for the equality of coefficients of variation from k populations. Statistics in Medicine 15:647-658.

Fieller, E. C. (1932). A numerical test of the adequacy of A. T. McKay's approximation. Journal of the Royal Statistical Society 95:699-702.

Forkman, J. and Verrill, S. (2008). The distribution of McKay's approximation for the coefficient of variation. Statistics 83 Probability Letters 78:10-14. 
Fung W. K. and Tsang T. S. (1998). A simulation study comparing tests for the equality of coefficients of variation. Statistics in Medicine 17:20032014 .

Gerig T. M. and Sen A. R. (1980). MLE in two normal samples with equal but unknown population coefficients of variation. Journal of the American Statistical Association 75:704-708.

Gupta R. C. and Ma S. (1996). Testing the equality of coefficients of variation in $\mathrm{k}$ normal populations. Communications in Statistics Theory and Methods 25:115-132.

Hartley H. O. (1950). The maximum F-ratio as a short cut test for heterogeneity of variance. Biometrika 37:308-312.

Iglewicz B. and Myers R. H. (1970). Comparison of approximations to the percentage points of the sample coefficient of variation. Technometrics 12:166-169.

Lohrding R. K. (1975). A two sample test of equality of coefficients of variation or relative errors. Journal of Statistical Computation and Simulation 4:31-36.

Marchand E. (1997). On moments of beta mixtures, the noncentral beta distribution, and the coefficient of determination. Journal of Statistical Computation and Simulation 59:161-178.

McKay, A. T. (1932). Distribution of the coefficient of variation and the extended 't' distribution. Journal of the Royal Statistical Society 95:695698 .

Miller G. E. (1991a). Asymptotic test statistics for coefficients of variation. Communications in Statistics - Theory and Methods 20:3351-3363.

Miller G. E. (1991b). Use of the squared ranks test to test for the equality of the coefficients of variation. Communications in Statistics - Simulation and Computation 20:743-750.

Miller G. E. and Feltz C. J. (1997). Asymptotic inference for coefficients of variation. Communications in Statistics - Theory and Methods 26:715726.

Nairy, K. S. and Rao, K. A. (2003). Tests of coefficients of variation of normal population. Communications in Statistics - Simulation and Computation 32:641-661. 
Nelson L. S. (1987). Upper 10\%, 5\% and $1 \%$ points of the maximum Fratio. Journal of Quality Technology 19:165-167.

Owen D. B. (1968). A survey of properties and applications of the noncentral t-distribution. Technometrics 10:445-478.

Pearson E. S. (1932). Comparison of A.T. McKay's approximation with experimental sampling results. Journal of the Royal Statistical Society 95:703-704.

Serfling, R. J. (1980) Approximation Theorems of Mathematical Statistics. New York: Wiley.

Shafer N. J. and Sullivan J. A. (1986). A simulation study of a test for the equality of the coefficients of variation. Communications in Statistics - Simulation and Computation 15:681-695.

Tian L. (2005). Inferences on the common coefficient of variation. Statistics in Medicine 24:2213-2220.

Tsui K. W. and Weerahandi S. (1989). Generalized $P$-values in significance testing of hypotheses in the presence of nuisance parameters. Journal of American Statistical Association 84:602-607.

Umphrey G. J. (1983). A comment on McKay's approximation for the coefficient of variation. Communications in Statistics - Simulation and Computation 12:629-635.

Vangel M. G. (1996). Confidence intervals for a normal coefficient of variation. The American Statistician 15:21-26.

Verrill S. and Johnson R. A. (2007). Confidence bounds and hypothesis tests for normal distribution coefficients of variation. Communications in Statistics - Theory and Methods 36:2187-2206.

Zeigler R. K. (1973). Estimators of coefficients of variation using $k$ samples. Technometrics 15:409-414. 